\title{
MAPEAMENTO DE SEMEADURAS DE SOJA (Glycine max (L.)Merr.) MEDIANTE DADOS MODIS/TERRA E TM/LANDSAT 5: UM COMPARATIVO
}

\author{
RUBENS A. C. LAMPARELLI ${ }^{1}$, WASTE M. O. DE CARVALHO ${ }^{2}$, \\ ERIVELTO MERCANTE ${ }^{3}$
}

\begin{abstract}
RESUMO: O objetivo deste trabalho foi comparar mapeamentos de semeadura da cultura da soja na região oeste do Paraná, realizados com imagens MODIS/Terra e TM/Landsat 5. Primeiramente, construiu-se máscara de referência, considerando seis imagens TM ao longo do ciclo da cultura, utilizando-se dos algoritmos Paralelepípedo e MaxVer com posterior análise visual. As imagens MODIS foram classificadas com o algorítimo Paralelepípedo, em quatro passagens referentes ao pico vegetativo. O desempenho das classificações foi avaliado por meio de Matrizes de Erros, calculadas pela análise de 100 pontos amostrais (soja ou não-soja), aleatoriamente distribuídos em cada um dos oito municípios da área de estudo. Os principais resultados mostraram que a Exatidão Global (EG) e o Índice Kappa (IK), que variaram entre 0,55 e 0,80, em ambos os sensores, são considerados bons a muito bons. Quando EG e IK dos sensores TM e MODIS foram comparados, não se encontrou diferença significativa. O mapeamento da soja utilizando o sensor MODIS produziu $70 \%$ de confiabilidade sob o ponto de vista do usuário. A principal conclusão é a viabilidade de mapear a soja pelo sensor MODIS com as vantagens de que as imagens MODIS têm melhor resolução temporal e são disponibilizadas gratuitamente na Internet.
\end{abstract}

PALAVRAS-CHAVE: monitoramento, matriz de erros, previsão de safras, imagens orbitais, culturas agrícolas.

\section{MAPPING OF SOYBEAN (Glycine max (L.) Merr.) CULTURE BY MODIS/TERRA AND TM/LANDSAT 5: A COMPARATIVE}

\begin{abstract}
The objective of this work was to compare the soybean crop mapping in the western of Parana State by MODIS/Terra and TM/Landsat 5 images. Firstly, it was generated a soybean crop mask using six TM images covering the crop season, which was used as a reference. The images were submitted to Parallelepiped and Maximum Likelihood digital classification algorithms, followed by visual inspection. Four MODIS images, covering the vegetative peak, were classified using the Parallelepiped method. The quality assessment of MODIS and TM classification was carried out through an Error Matrix, considering 100 sample points between soybean or not soybean, randomly allocated in each of the eight municipalities within the study area. The results showed that both the Overall Classification (OC) and the Kappa Index (KI) have produced values ranging from 0.55 to 0.80 , considered good to very good performances, either in TM or MODIS images. When OC and KI, from both sensors were compared, it wasn't found no statistical difference between them. The soybean mapping, using MODIS, has produced $70 \%$ of reliance in terms of users. The main conclusion is that the mapping of soybean by MODIS is feasible, with the advantage to have better temporal resolution than Landsat, and to be available on the internet, free of charge.
\end{abstract}

KEYWORDS: monitoring, error matrix, yield estimate, orbital images, crops.

\footnotetext{
${ }^{1}$ Eng $^{\mathrm{o}}$ Agrícola, Pesquisador, CEPAGRI/UNICAMP, Cidade Universitária, s/n, Campinas - SP, Fone: (0XX19) 3251.2465, rubens@cpa.unicamp.br

${ }^{2}$ Estatística, Graduanda, Cidade Universitária, s/n, Campinas - SP, Fone: (0XX19) 3251.2465, wasteoc@ig.com.br

${ }^{3}$ Eng $^{\mathrm{O}}$ Agrícola, Doutorando, FEAGRI/UNICAMP, Cidade Universitária, s/n, Campinas - SP, Fone: (0XX19) 3251.2465, erivelto@agr.unicamp.br

Recebido pelo Conselho Editorial em: 10-4-2007
}

Aprovado pelo Conselho Editorial em: 10-3-2008 


\section{INTRODUÇÃO}

O setor do agronegócio, como um todo, envolve mais de 1/3 do PIB brasileiro (MELO, 2004). Ele produz alimentos, gera mais de 16 milhões de empregos, além de diversificar e de aumentar consideravelmente as exportações. Nesse sentido, a estimativa de safra assume importância fundamental.

Dois são os pilares que sustentam a previsão de safras de um país: o primeiro é a área ocupada pela cultura, e o segundo, a produtividade da mesma.

Nesses dois pilares mencionados, o Sensoriamento Remoto vem contribuindo de forma decisiva na geração de informações, tal como pode ser observado em trabalhos como MASS (1988), GROTEN (1993), LOBELL et al. (2003), DORAISWAMY et al. (2004), KASTENS et al. (2005). Apesar desse relativo sucesso, ainda é possível identificar a necessidade de aprimoramentos quanto às resoluções temporal (minimizar a ocorrência de cobertura de nuvens) e espacial. Alguns sensores apresentam alta resolução espacial, mas baixa resolução temporal, enquanto outros não possuem adequada resolução espacial, mas, em compensação, apresentam ótima resolução temporal, com passagens diárias (MODIS/Terra-Aqua, AVHRR/NOAA), quando são utilizados no monitoramento de culturas. Muitos trabalhos mostram a utilização e a integração de vários sensores para monitorar culturas ou suas variáveis.

TUCKER et al. (2005) apresentaram um trabalho de construção de banco de dados por valores de NDVI, calculados a partir de imagens NOAA/AVHRR, os quais foram comparados com valores de NDVI, calculados a partir dos sensores MODIS e SPOT VEGETATION. O trabalho evidenciou a possibilidade de utilização de diferentes sensores no monitoramento de culturas para complementar uma série histórica do sensor AHVRR/NOAA, na eventual perda de informações.

SEE \& FRITZ (2006) mostraram a utilidade da integração de dados de vários sensores na geração de mapas de uso da terra em escala global. Nesse trabalho, foram utilizadas imagens dos sensores MODIS, VEGETATION, TM e ETM+. A integração proporcionou detalhamento do uso da terra em escalas regional e nacional, criando um conjunto de dados com diferentes escalas. STERN et al. (2001) utilizaram dados do sensor TM/Landsat para aumentar a acurácia da classificação do trigo de inverno no Estado de Dakota do Norte/USA, em imagens AVHRR. Os autores constataram que esse método aumentou a eficácia da classificação, sendo que os resultados de área estimada pelo sensor AVHRR aproximaram-se dos dados do "United States Department of Agriculture" (USDA), para o ano de 1994.

KASTENS et al. (2005) descrevem metodologia para estimar a produtividade de culturas, em nível regional, em que a confecção de uma máscara que contemple culturas em geral, sem especificar qual, chamada de máscara de correlação de produtividade ("yield-correlation masking"), pode melhorar a estimativa de produtividade. O procedimento requer uma série temporal de imagens, o correspondente valor regional de produtividade e envolve a correlação histórica dos valores dos "pixels" das imagens com os valores históricos regionais de produtividade.

A bibliografia também relata que, para cada mapa de uso ou de mudanças de uso, algumas ferramentas específicas são utilizadas para medir a qualidade desses mapas. Uma das possibilidades mais utilizadas é a matriz de erros. CONGALTON et al. (1983), STORY \& CONGALTON (1986) e ROSENFIELD \& FITZPATRICK-LINS (1986) utilizaram a matriz de erros para gerar o índice de qualidade global e o coeficiente de concordância como medidas de acurácia em classificações temáticas.

MERCANTE et al. (2006) utilizaram a matriz de erros para ponderar a estimativa de áreas obtidas por classificação supervisionada, por meio da relação entre a área de cada classe e o total de área analisada. O método foi comparado com os dados oficiais, e as diferenças variaram entre $-4 \% \mathrm{e}$ 19\%. Da mesma forma, RUDORFF et al. (2007) avaliaram imagens MODIS para a classificação e a estimativa de área de soja no Rio Grande do Sul, por meio do método de superfície de resposta espectro-temporal. Eles utilizaram máscara das áreas de soja oriunda de imagens TM/Landsat, e os 
resultados foram avaliados pela Estatística Kappa. Os resultados mostraram que a área total foi subestimada em 6,6\%. Para as regiões de latifúndios e minifúndios, a área de soja foi superestimada em $8 \%$ e subestimada em $43,4 \%$, respectivamente.

Este trabalho teve como objetivo comparar mapeamentos de semeaduras de soja (Glycine max (L.) Merr.) a partir de imagens MODIS/TERRA e TM/LANDSAT, no oeste do Paraná, durante a safra 2003/2004.

\section{MATERIAL E MÉTODOS}

\section{Métodos}

A área de estudo foi composta por oito municípios do oeste do Estado do Paraná, alocados dentro da cena 223/077 do Landsat 5/TM: Assis Chateaubriand, Cascavel, Mamborê, Maripá, Palotina, Rancho Alegre d'Oeste, Toledo e Ubiratã (Figura 1).

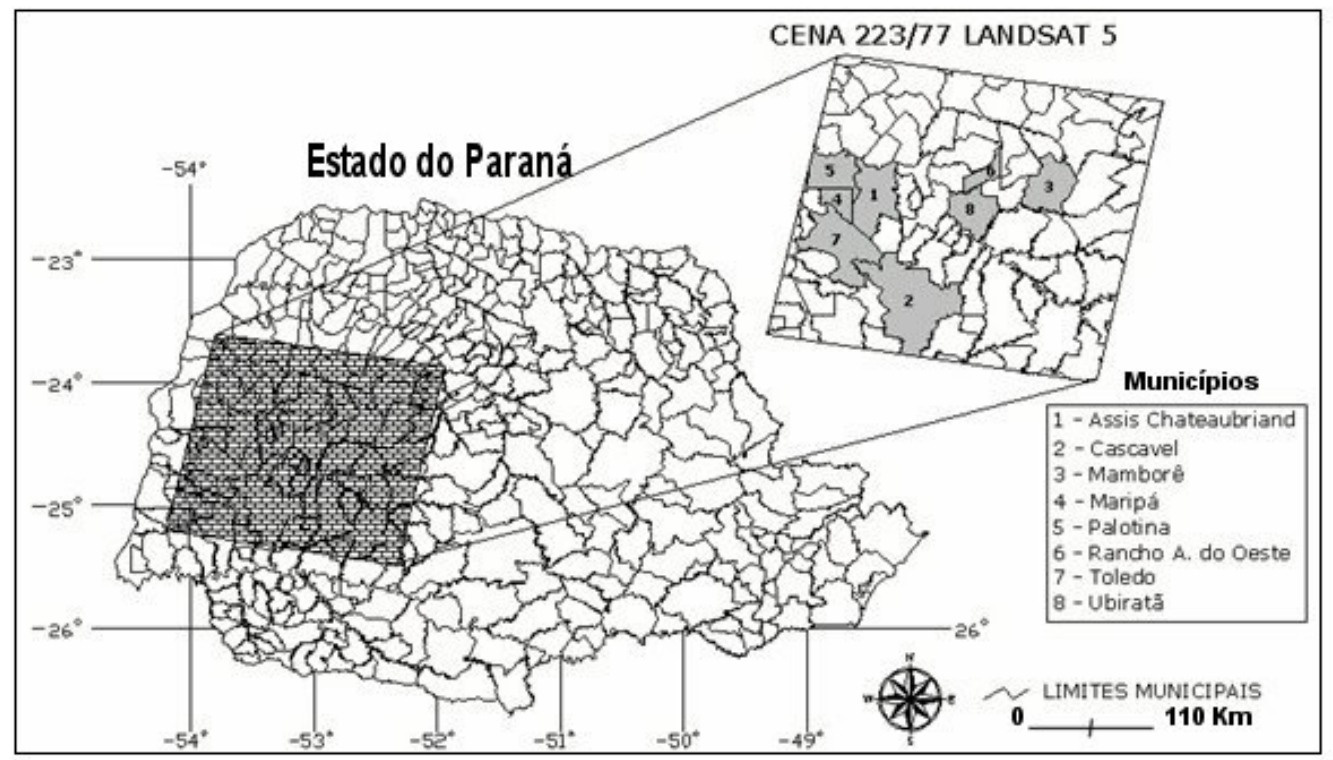

FIGURA 1. Localização da área de estudos, no oeste do Estado do Paraná, em relação à cena TM/Landsat 5 utilizada. Location of the study area, in the western region of Paraná

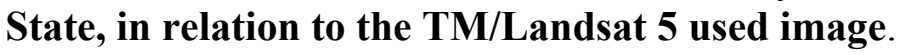

Foram utilizadas imagens do Sensor MODIS da safra 2003/2004, especificamente o produto MOD13Q1 de Índice de Vegetação, com resolução radiométrica de 16 bits, resolução espacial de $250 \mathrm{~m}$ e composição de 16 dias (http://modis.gsfc.nasa.gov/). Foram utilizadas imagens de quatro datas, correspondentes ao pico vegetativo: 19-12-2003, 1ํ-1-2004, 17-1-2004 e 2-2-2004.

A máscara TM/Landsat 5 foi utilizada como referência, sendo gerada a partir das imagens dos dias 21-11-2003, 7-12-2003, 23-12-2003, 24-1-2004, 9-2-2004 e 25-2-2004. Os vetores que representam o mapeamento da cultura da soja nas imagens do sensor TM/Landsat-5 foram utilizados para aplicar uma máscara sobre a imagem do MODIS. Para o processamento das imagens, foram utilizados o aplicativo ENVI (RSI, 2003) e o programa IDRISI Kilimanjaro (EASTMAN, 2003).

A primeira etapa de processamento das imagens foi o georreferenciamento; a seguir, foi executada a transformação radiométrica, ou seja, a conversão do Número Digital (ND) para valores de reflectância aparente (no topo da atmosfera), conforme CHANDER \& MARKHAN (2003). Essa estratégia foi adotada, ainda segundo CHANDER \& MARKHAN (2003), para diminuir a variabilidade das respostas intercenas, corrigir as imagens para o efeito de diferenças do ângulo zenital solar (devido às épocas diferentes de tomada das imagens) e compensar as diferenças de irradiância solar no topo da atmosfera. 
Com base em composições coloridas RGB-453, falsa cor, foram coletadas as amostras de "pixels" que correspondiam à cultura da soja, tomando como base a evolução de uma área agrícola ocupada com a cultura de soja durante a safra monitorada, situada próxima ao Município de Cascavel - PR. Em seguida, foram executadas as classificações supervisionadas por meio dos algoritmos de classificação Paralelepípedo e Máxima Verossimilhança, nas imagens de reflectância aparente. Eles foram utilizados de forma complementar, pois cada um deles apresentou limitações. Foram também realizadas inspeções visuais posteriomente às classificações. Nas classificações, foram utilizadas as bandas 3; 4; 5 e 7, como forma de minimizar os efeitos atmosféricos, pois são menos influenciadas pelos efeitos de absorção e de espalhamento dos aerossóis e vapor d'água presentes na atmosfera. De posse das classificações, para as seis imagens, a máscara final foi encontrada, realizando a operação booleana $O R$ de todas elas, pois para cada data é identificada parte da área com a cultura que, por apresentar épocas de semeadura diferentes, só será mapeada pelo sensor, também em diferentes passagens. Dessa forma, a operação booleana garante que as áreas mapeadas em uma data, e não em outras, sejam contempladas na imagem final.

Para as imagens MODIS, foram escolhidas quatro imagens referentes ao pico do ciclo vegetativo da soja, que, na região, varia de 15 de janeiro a 15 de fevereiro, expressas em valores de NDVI, composição de 16 dias. Para as imagens MODIS, foi realizada a classificação com o algoritmo Paralalepípedo. Esse foi o algoritmo mais apropriado, pois os valores de NDVI variavam muito pouco, e o classificador como Máxima Verossimilhança classificava todos os "pixels" em uma única classe. As amostras foram obtidas por meio da máscara elaborada a partir de imagens do sensor TM/Landsat 5 (resolução espacial de $30 \mathrm{~m}$ ) superposta às imagens MODIS.

A qualidade final das classificações das áreas de soja, tanto para o sensor TM quanto para o sensor MODIS, foi avaliada pelas métricas de Exatidão Global e Índice Kappa. Foram utilizados 100 pontos amostrais (LUIZ, 2005) distribuídos aleatoriamente por município, nos oito escolhidos, e com eles foram construídas as Matrizes de Erros. Esses pontos foram verificados no campo por técnicos da Conab - Companhia Nacional de Abastecimento (FIGUEIREDO, 2007), e organizados na forma de planilhas, considerando duas classes no ponto verificado - ou era soja (independentemente do estágio) ou não era soja (qualquer outra coisa - mata, pasto).

Com as comparações mapa temático/referência, foram elaboradas as Matrizes de Erro e calculados os valores de Kappa e da Exatidão Global assim como os erros e a precisão sob os pontos de vista do produtor e do usuário, por município. Essas métricas permitem avaliar melhor a classificação final das áreas de soja. Para avaliar a qualidade das estatísticas, foram escolhidos os valores de referência dados por LANDIS \& KOCH (1977).

\section{Testes de comparações entre coeficientes de exatidão}

Para realizar a comparação entre os mapeamentos obtidos por meio dos sensores Modis e TM, optou-se por comparar os coeficientes de exatidão obtidos pelas classificações. Sejam Exatidão Global (Po) e Índice Kappa (K), calculados pelas eqs. (1) e (2), respectivamente (CONGALTON \& GREEN, 1999):

$$
\text { Po }=\frac{\sum_{i=1}^{r} x_{i i}}{N}
$$

em que,

$\mathrm{N}$ - número total de unidades amostrais;

$\mathrm{x}_{\mathrm{ii}}$ - elementos da diagonal principal;

$\mathrm{r}$ - número de linhas na Matriz de Erros, e

$\mathrm{i}, \mathrm{j}$ - linhas e colunas da Matriz, respectivamente. 


$$
\mathrm{K}=\frac{\mathrm{N} \sum_{\mathrm{i}=1}^{\mathrm{r}} \mathbf{X}_{\mathrm{ii}}-\sum_{\mathrm{i}=1}^{\mathrm{r}} \mathbf{X}_{\mathrm{i}+\mathrm{X}} \mathbf{X}_{+\mathrm{i}}}{\mathrm{N}^{2}-\sum_{\mathrm{i}=1}^{\mathrm{r}}\left(\mathrm{X}_{\mathrm{i}+} \mathbf{X}_{+\mathrm{i}}\right)}
$$

em que,

$\mathrm{N}$ - número total de unidades amostrais;

$\mathrm{r}$ - número de linhas da Matriz de Erros;

xii - observação da i-ésima linha e i-ésima coluna;

$\mathrm{xi}+$ - total marginal da linha i, e

$\mathrm{x}+\mathrm{i}$ - total marginal da coluna $\mathrm{i}$.

A Exatidão Global (Po) segue uma distribuição de probabilidade Binomial. Quando o número de "pixels" usados para a construção da Matriz de Erros é grande, o Teorema Central do Limite permite aproximar a distribuição Binomial à distribuição Normal.

Para usar a aproximação Normal nos testes de diferença entre classificações de imagens, segundo MA \& REDMOND (1995), é preciso que as seguintes suposições sejam satisfeitas:

- as amostras devem ser selecionadas independentemente;

- as categorias de cobertura da terra devem ser independentes e mutuamente exclusivas, e

- as classificações devem ser processadas independentemente.

Com essas suposições garantidas, tem-se que, sob a hipótese de igualdade entre dois coeficientes de exatidão vindos de classificações diferentes $(\mathrm{C} 1=\mathrm{C} 2)$, a estatística do teste é:

$\mathrm{Z}=\frac{\mathrm{C}_{1}-\mathrm{C}_{2}}{\sqrt{\sigma_{1}^{2}+\sigma_{2}^{2}}} \sim \mathrm{N}(0,1)$

Para a Exatidão Global, $C=$ Po, a estimativa da variância é,

$\hat{\sigma}_{\mathrm{Po}}^{2}=\frac{\mathrm{P}_{0}\left(1-\mathrm{P}_{0}\right)}{\mathrm{N}}$

Para o Índice Kappa, $\mathrm{C}=\mathrm{K}$, a estimativa da variância é,

$$
\hat{\sigma}_{\mathrm{K}}^{2}=\frac{\mathrm{P}_{0}\left(1-\mathrm{P}_{0}\right)}{\mathrm{N}(1-\mathrm{Pe})^{2}} \quad \text { e } \quad \mathrm{Pe}=\sum_{\mathrm{i}=1}^{\mathrm{r}} \frac{\mathrm{x}_{\mathrm{i}+} \mathrm{x}_{+\mathrm{i}}}{\mathrm{N}^{2}}
$$

em que,

$\mathrm{P}_{\mathrm{o}}$ - Exatidão Global (probabilidade a posteriori);

Pe - concordância randômica (probabilidade a priori);

$\mathrm{N}$ - número total de unidades amostrais;

$\mathrm{r}$ - número de linhas da Matriz de Erros;

xi+ - total marginal da linha i, e

$\mathrm{x}+\mathrm{i}$ - total marginal da coluna $\mathrm{i}$.

O teste de igualdade foi realizado com nível de 5\% significância.

Por final, a área identificada de soja foi comparada com aquela fornecida pelo IBGE como mais um parâmetro de avaliação da qualidade do mapeamento. A comparação seguiu o método COCHRAN (1977), utilizando, para estimar as variáveis agrícolas obtidas por amostragem, a expansão direta das informações amostradas, neste caso, a área.

A estimativa por expansão direta utiliza apenas os dados coletados no campo, a respeito das diferentes coberturas vegetais para os pontos amostrais da CONAB. Esse método, em que se cria 
um painel amostral de "pixels" que estão inseridos em limites compostos de classes de uso, permite rápida avaliação do volume de área de certa classe de uso do solo.

Baseado nisso, a estimativa por expansão direta consiste na multiplicação da proporção de pontos de cada cultura em um município pela área municipal, dada pela eq.(6) (EPIPHANIO et al., 2002):

$$
\mathrm{Z}_{\mathrm{hc}}=\mathrm{v}_{\mathrm{h}} \mathrm{n}_{\mathrm{hc}}
$$

em que,

$\mathrm{Z}_{\mathrm{hc}}$ - área estimada da cultura c no município h;

$\mathrm{v}_{\mathrm{h}}$ - fator de expansão para um ponto, e

$\mathrm{n}_{\mathrm{hc}}$ - número de pontos amostrais da categoria $\mathrm{c}$.

O fator de expansão é dado pela eq.(7):

$$
\mathrm{v}_{\mathrm{h}}=\mathrm{a}_{\mathrm{h}} / \mathrm{n}_{\mathrm{h}}
$$

em que,

$\mathrm{a}_{\mathrm{h}}$ - área total do município h, e

$\mathrm{n}_{\mathrm{h}}$ - número de pontos amostrais no município $\mathrm{h}$.

\section{RESULTADOS E DISCUSSÃO}

A classificação de soja na máscara oriunda das imagens Landsat (Figura 2) produziu os resultados de Exatidão Global e Índice Kappa, para 100 pontos distribuídos em cada município da área de estudo, mostrados na Tabela 1.

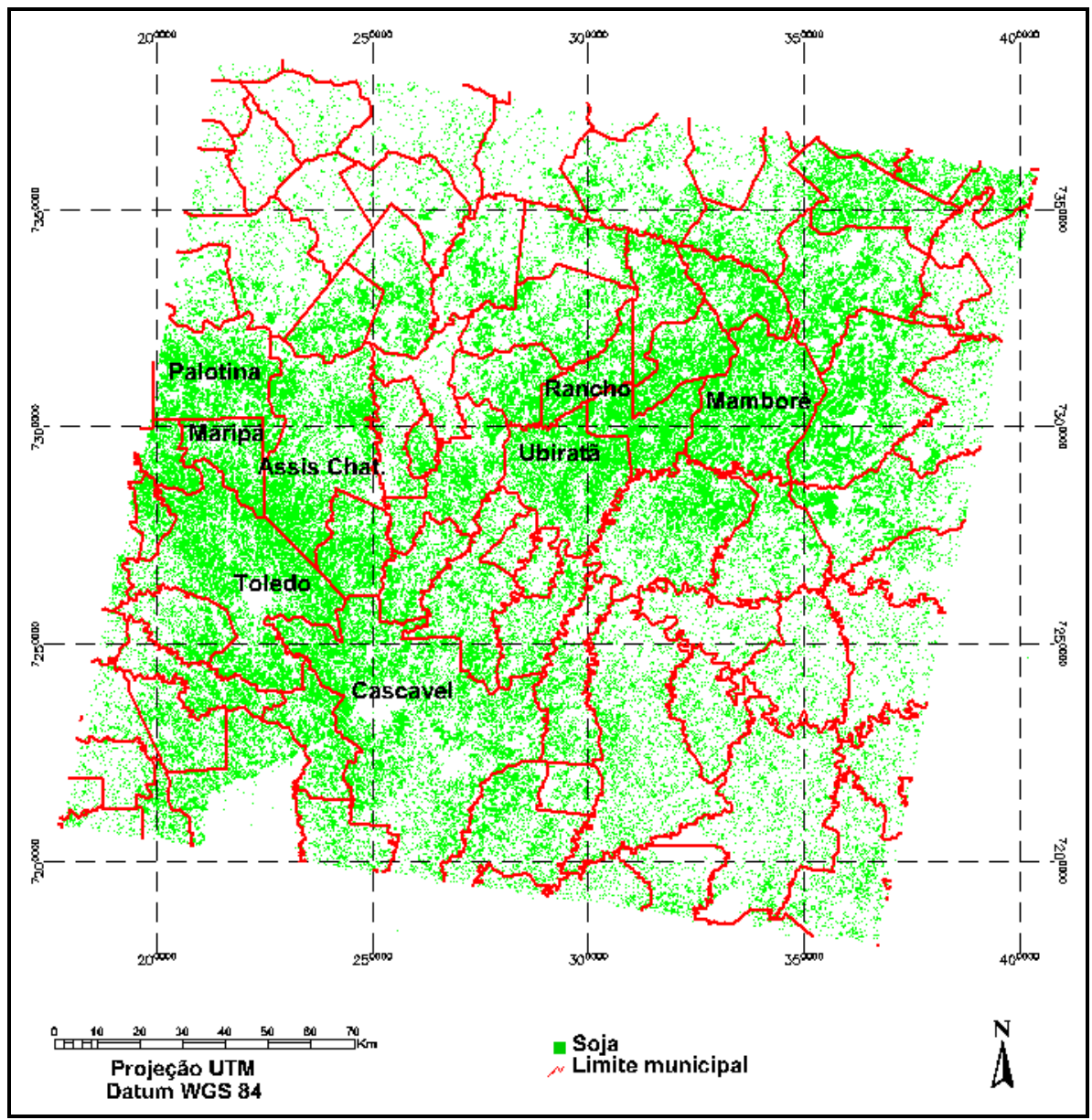

FIGURA 2. Máscara de soja oriunda das imagens TM/Landsat 5. Soybean crop area mask derived from TM/Landsat 5 digitally classified images. 
TABELA 1. Avaliação das classificações das imagens dos sensores MODIS e TM. Classification evaluation using MODIS and TM images.

\begin{tabular}{cccccc}
\hline \multirow{2}{*}{ Município } & \multicolumn{2}{c}{ MODIS } & \multicolumn{2}{c}{ TM } & \multirow{2}{*}{ Avaliação } \\
\cline { 2 - 5 } & Exatidão Global & Kappa & Exatidão Global & Kappa & \\
\hline Assis Chateaubriand & 0,740 & 0,737 & 0,710 & 0,708 & MB/MB \\
Cascavel & 0,680 & 0,672 & 0,810 & 0,808 & MB/MB \\
Mamborê & 0,560 & 0,542 & 0,800 & 0,799 & B/MB \\
Maripá & 0,620 & 0,609 & 0,660 & 0,650 & MB/MB \\
Palotina & 0,535 & 0,510 & 0,646 & 0,635 & B/MB \\
Rancho Alegre do Oeste & 0,705 & 0,700 & 0,768 & 0,767 & MB/MB \\
Toledo & 0,640 & 0,629 & 0,750 & 0,746 & MB/MB \\
Ubiratã & 0,620 & 0,610 & 0,790 & 0,789 & MB/MB \\
\hline
\end{tabular}

Muito Boa (MB), Boa (B).

De acordo com os valores de referência de LANDIS \& KOCH (1977), a qualidade das classificações no MODIS e no TM, apresentadas pela TABELA 1, estão na faixa de Boa a Muito Boa. Como era de se esperar, a identificação nas imagens MODIS ficou um pouco aquém das classificações das imagens TM.

TABELA 2. Diferença entre as áreas estimada (Imagem de satélite) e Oficial. Difference between estimated areas from satellite images and official figures.

\begin{tabular}{ccccc}
\hline Município & $\begin{array}{c}\text { Número de Pontos } \\
\text { com Soja Certa }\end{array}$ & $\begin{array}{c}\text { Área de Soja por } \\
\text { Município - Estimada } \\
(\text { Imag. })-\left(\mathrm{km}^{2}\right)\end{array}$ & $\begin{array}{c}\text { Área de Soja } \\
\text { Oficial (Of)* } \\
\left(\mathrm{km}^{2}\right)\end{array}$ & $\begin{array}{c}\text { Diferença de Área } \\
\text { (Of.-Imag)/Of. } \\
(\%)\end{array}$ \\
\hline Assis Chateaubriand & 52 & $48.724,11$ & 72.000 & 32,32 \\
Cascavel & 23 & $82.434,42$ & 82.000 & $-0,53$ \\
Mamborê & 43 & $46.961,46$ & 54.000 & 13,00 \\
Maripá & 31 & $16.324,11$ & 21.500 & 24,07 \\
Palotina & 37 & $34.002,09$ & 43.300 & 21,47 \\
Rancho Alegre do Oeste & 59 & $14.565,33$ & 15.400 & 5,42 \\
Toledo & 37 & $66.565,89$ & 68.600 & 2,97 \\
Ubiratã & 47 & $36.069,84$ & 51.200 & 29,55 \\
\hline
\end{tabular}

*SEAD/DERAL (2005) - atualização 20-1-2005.

Pode-se notar, na Tabela 2, que, em alguns municípios, a diferença atingiu quase $30 \%$; em outro, a diferença foi muito pequena. Algumas considerações podem ser feitas. Primeira, a própria limitação apresentada pelos classificadores booleanos utilizados. A estratégia foi fazer uso, de forma complementar, dos dois classificadores com vistas a aumentar o poder de identificação da cultura.

Os erros apresentados pelos classificadores podem ser minimizados utilizando-se da metodologia proposta por JUPP (1989), que pondera os valores de área pelos erros encontrados na matriz. Esse procedimento foi testado por MERCANTE et al. (2006) para imagens Landsat em duas safras, para soja, no oeste do Paraná. O erro foi distribuído pelos valores de áreas dos oito municípios. Independentemente do método, os autores encontraram dificuldade no momento de comparar os valores a uma referência. O problema está na escolha da referência que seja confiável e representativa da realidade. XIAO et al. (2002) observaram, em escala municipal, diferenças entre os valores de área estimada por satélites com os valores das estatísticas oficiais, que ora subestimavam (7\%), ora superestimavam (92\%). Nesse contexto, LUIZ (2005) e IPPOLITIRAMILO et al. (2003) fazem menção a esse problema, pois encontraram dados conflitantes com os dados oficiais.

Outro fator que certamente implica diminuição da precisão da avaliação de área está no tamanho do "pixel" do MODIS (250 x 250 m). DORAISWAMY et al. (2004) mencionaram que o 
sensor MODIS é adequado para monitorar áreas maiores que 25 ha. Na região oeste do Paraná, a maioria das propriedades possui área maior ou igual a 25 ha. Alguns trabalhos mostram que, em média, as áreas de produtores de soja no oeste do Paraná são de 30 ha, com tendência de crescimento devido ao efeito de escala (SCHNEPF et al., 2001; FLASKERUD, 2003; BOZZA \& GIOVANI, 2004). Portanto, apesar de o trabalho aqui apresentado não analisar esse quesito, acredita-se que existe um erro embutido, não quantificado nesta análise. Por outro lado, as áreas são praticamente contíguas, o que favorece a identificação por esse tipo de sensor.

A Exatidão Global mede a precisão total do mapeamento, que, neste caso, foi calculada por Município. Para medir a precisão de cada categoria, individualmente (soja e não-soja), usou-se o erro do ponto de vista do produtor e do usuário. Na Tabela 3, mostra-se o erro e a precisão para a categoria de interesse, soja.

TABELA 3. Erro e precisão do ponto de vista do produtor e do usuário para as Matrizes de Erros construídas a partir de imagens MODIS. Producer's and user's error and accuracy, based on error matrices derived from MODIS images.

\begin{tabular}{ccccc}
\hline \multirow{2}{*}{ Município } & \multicolumn{2}{c}{ Ponto de Vista do Produtor } & \multicolumn{2}{c}{ Ponto de Vista do Usuário } \\
\cline { 2 - 5 } & Erro & Precisão & Erro & Precisão \\
\hline Assis Chateaubriand & 0,088 & 0,912 & 0,288 & 0,712 \\
Cascavel & 0,452 & 0,547 & 0,361 & 0,639 \\
Mamborê & 0,232 & 0,768 & 0,419 & 0,581 \\
Maripá & 0,475 & 0,525 & 0,244 & 0,756 \\
Palotina & 0,448 & 0,552 & 0,315 & 0,685 \\
Rancho Alegre do Oeste & 0,092 & 0,908 & 0,314 & 0,686 \\
Toledo & 0,275 & 0,725 & 0,373 & 0,627 \\
Ubiratã & 0,161 & 0,839 & 0,382 & 0,618 \\
\hline
\end{tabular}

Para o município de Assis Chateaubriand, por exemplo, tem-se que a exatidão global foi de $74,0 \%$ (Tabela 1). Porém, do ponto de vista do produtor, a precisão foi de $91,2 \%$; e do ponto de vista do usuário, a precisão foi de $71,2 \%$. Assim, embora $91,2 \%$ das áreas com a cultura de soja tenham sido identificadas como soja, apenas $71,2 \%$ das áreas identificadas como sendo soja na imagem, era, de fato, cultura de soja. Esses resultados são semelhantes aos obtidos por RUDORFF et al. (2007), no Rio Grande do Sul, que encontraram Exatidão Global de 76,17\%. Apesar do erro de $30 \%$, do ponto de vista do usuário, em locais onde não se tem informação, essa metodologia poderia ser aplicada com bons resultados.

\section{Testes de igualdade}

Para verificar se as diferenças entre os índices de exatidão das classificações foram estatisticamente significativas, realizaram-se os testes de hipótese usando a aproximação Normal da estatística $Z$, sendo a confiança utilizada de $95 \%(\alpha=5 \%)$. Os resultados para a Exatidão Global estão na Tabela 4 e, para o índice Kappa, na Tabela 5.

TABELA 4. Teste de hipótese para diferença da Exatidão Global entre MODIS e TM. Hypothesis test for overall accuracy between MODIS and TM data.

\begin{tabular}{cccccc}
\hline Município & Diferença & Desvio-Padrão & $\mathrm{Z}$ & P-valor & Resultado para a Diferença \\
\hline Assis Chateaubriand & 0,03 & 0,063 & 0,475 & 0,631 & Não-significativa \\
Cascavel & 0,13 & 0,061 & 2,133 & 0,033 & Significativa \\
Mamborê & 0,24 & 0,064 & 3,765 & 0,000 & Significativa \\
Maripá & 0,04 & 0,068 & 0,590 & 0,555 & Não-significativa \\
Palotina & 0,11 & 0,069 & 1,608 & 0,107 & Não-significativa \\
Rancho Alegre do Oeste & 0,06 & 0,062 & 1,017 & 0,308 & Não-significativa \\
Toledo & 0,11 & 0,065 & 1,702 & 0,089 & Não-significativa \\
Ubiratã & 0,17 & 0,063 & 2,683 & 0,007 & Significativa \\
\hline
\end{tabular}


TABELA 5. Teste de hipótese para diferença do índice Kappa entre MODIS e TM. Hypothesis test between MODIS and TM Kappa Agreement Index.

\begin{tabular}{cccccc}
\hline Município & Diferença & Desvio-Padrão & $\mathrm{Z}$ & \multicolumn{2}{c}{ P-valor Resultado para a Diferença } \\
\hline Assis Chateaubriand & 0,03 & 0,130 & 0,226 & 0,818 & Não-significativa \\
Cascavel & 0,13 & 0,129 & 1,055 & 0,289 & Não-significativa \\
Mamborê & 0,26 & 0,138 & 1,871 & 0,061 & Significativa \\
Maripá & 0,04 & 0,134 & 0,307 & 0,757 & Não-significativa \\
Palotina & 0,12 & 0,139 & 0,897 & 0,368 & Não-significativa \\
Rancho Alegre do Oeste & 0,07 & 0,156 & 0,431 & 0,667 & Não-significativa \\
Toledo & 0,12 & 0,130 & 0,908 & 0,363 & Não-significativa \\
Ubiratã & 0,18 & 0,137 & 1,308 & 0,191 & Não-significativa \\
\hline
\end{tabular}

Para os dois coeficientes, Exatidão Global e índice Kappa, a maioria dos testes resultou em diferença não-significativa, indicando que, nesses casos, as classificações conduzidas a partir dos sensores MODIS e TM não foram significativamente diferentes.

Do município de Assis Chateaubriand, por exemplo, o resultado do teste para a Exatidão Global foi de diferença não-significativa, com p-valor igual a 0,818 , que indicou que as classificações oriundas dos dados dos dois sensores foram similares (quanto mais próximo o p-valor está de 1, menor é a evidência contra a hipótese de igualdade). Para o município de Mamborê, o teste resultou em diferença significativa, nos dois coeficientes de exatidão. Porém, para a Exatidão Global, o p-valor foi igual a zero (quanto mais próximo o p-valor está de zero, maior é a evidência contra a hipótese de igualdade). Para a Estatística Kappa, o p-valor foi de 0,061, que está muito próximo do nível de significância do teste.

\section{CONCLUSÕES}

A classificação realizada, utilizando imagens TM/Landsat 5, apresentou bons resultados na Exatidão Global e Índice Kappa, com valores em torno de 0,6 a 0,8.

A classificação realizada, utilizando imagens MODIS, apresentou, em termos absolutos, bons resultados $(0,5$ a 0,7$)$, mas, relativamente, foi inferior ao resultado encontrado com imagens TM.

Não houve diferença de qualidade na identificação da cultura da soja avaliada através da Matriz de Erros entre os dois sensores.

Foi possível utilizar o sensor MODIS para mapear a cultura de soja com grau de confiabilidade em torno de $70 \%$, sob o ponto de vista do usuário.

Por apresentar alta resolução temporal, o MODIS é bastante apropriado, em relação ao sensor TM, para mapear a cultura de soja.

\section{AGRADECIMENTOS}

Ao CNPq, pelo financiamento das bolsas de Pibic e Doutorado do segundo e do terceiro autores, respectivamente. Aos assessores, pelas valiosas sugestões e críticas durante a elaboração deste artigo.

\section{REFERENCIAS}

BOZZA G.M.; DIGIOVANI, M.S. Crescimento do PIB dos EUA estimado em 4,7\% em 2004. Boletim Informativo n. 846, dezembro de 2004. FAEP - Federação da Agricultura do Estado do Paraná. 2004. 7 p. Disponível em: www.faep.com.br/boletim/bi846/pag6bi846.htm. Acesso em: 27 jul. 2007. 
CHANDER, G.; MARKHAM, B. Revised Landsat 5/TM radiometric calibration procedures and postcalibration Dynamic Ranges. IEEE Transactions on Geosciense and Remote Sensing, New York, v.41, n.11, p.2.764-77, 2003.

CONGALTON, R.G.; GREEN, K. Assessing the accuracy of remotely sensed data: principles and pratices. Washington: Lewis Publishers, 1999. 137 p.

CONGALTON, R.G.; ODERWALD, R.G.; MEAD, R.A. Assessing Landsat classification accuracy using discrete multivariate analysis Statistical Techniques. Photogrammetric Engineering and Remote Sensing, Reston, v.49, n.12, p.1.671-8, 1983.

COCHRAN, W.G. Sampling techniques. 3.ed. New York: John Wiley, 1977. 413 p.

DORAISWAMY, P.C.; HATFIELD, J.L.; JACKSON, T.J.; AKHMEDOV, B.; PRUEGER, J.; STERN, A. Crop condiction and yield simulations using Landsat and MODIS. Remote Sensing of Environment, New York, v. 92, p.548-59, 2004.

EASTMAN, J.R. Idrisi Kilimanjaro: guide to GIS and image processing. Worcester: Clark Laboratory/Clark Labs/Clark University, 2003. 328 p.

EPIPHANIO, J.C.N.; LUIZ, A.J.B.; FORMAGGIO, A.R. Estimativa de áreas agrícolas municipais utilizando sistema de amostragem simples sobre imagens de satélite. Bragantia, Campinas, v.61, n.2, p.187-97, 2002.

FLASKERUD, G. Brazil's production and impact soybean. Fargo: North Dakota State University, 2003. 16 p. Disponível em: www.ag.ndsu.edu/pubs/plantsci/rowcrops/eb79.pdf. Acesso em: 27 jul. 2007.

FIGUEIREDO, D.C. Projeto GeoSafras - aprimoramento metodológico das estimativas de safras. In: SIMPÓSIO BRASILEIRO DE SENSORIAMENTO REMOTO, 13., 2007, Florianópolis. Anais... São José dos Campos: INPE, 2007. p.191-5.

GROTEN, S.M.E. NDVI-Crop monitoring and early yield assessment of Burkina Faso. International Journal of Remote Sensing, London, v.14, n.8, p.1.495-15, 1993.

IPPOLITI-RAMILO, G.A.; EPIPHÂNIO, J.C.N.; SHIMABUKURO, Y.E. Landsat 5 thematic mapper data for pre-planting crop area evaluation in tropical countries. International Journal of Remote Sensing, London, v.24, n.7, p.1.521-34, 2003.

JUPP, D.L.B. The stability of global estimates from confusion matrices. International Journal of Remote Sensing, London, v.10, n.9, p.1.563-9, 1989.

KASTENS, J.H.; KSTENS, T.L.; KSTENS, D.L.A.; PRICE, K.P.; MARTINKO, E.A.; LEE, R.Y. Image masking for crop forecasting using AVHRR NDVI time series imagery. Remote Sensing of Enviroment, New York, v.99, n.3, p.341-56, 2005.

LANDIS, R.; KOCH, G.G. The measurement of observer agreement for categorical data.

Biometrics, Texas, v.33, n.1, p.159-74, 1977.

LOBELL, D.B.; ASNER, J.I.; ORTIZ-MONASTERIO; BENNING, T.L. Remote sensing of regional crop production in the Yaqui Valley, Mexico: estimates and uncertainties. Agriculture, Ecosystems and Environment, Amsterdam, v.94, n.2, p.205-20, 2003.

LUIZ, A.J.B. Estatísticas agrícolas por amostragem auxiliadas pelo sensoriamento remoto. In: SIMPÓSIO BRASILEIRO DE SENSORIAMENTO REMOTO, 12., 2005, Goiânia. Anais... São José dos Campos: INPE, 2007. p.181-8.

MA, Z.; REDMOND, R.L. Tau coefficients for accuracy assessment of classification of remote sensing data. Photogrammetric Engineering and Remote Sensing, Reston, v.61, n.4, p.63-152, 1995.

MASS, S.J. Using satellite data to improve model estimates of crop yield. Agronomy Journal, Madison, v.80, n.4, p.655-62, 1988. 
MELO, D.R.G.A. Dados recentes do agronegócio brasileiro. Revista da UFG, v.7, n.1, junho 2004. Disponível em: www.proec.ufg.br. Acesso em: 3 mar. 2006.

MERCANTE, E.; ANTUNES, J.F.G.; LAMPARELLI, R.A.; ROCHA, J.V. Estimativa de área da cultura de soja com imagens Landsat 5/TM por meio de matriz de erros. In: CONGRESSO BRASILEIRO DE ENGENHARIA AGRÍCOLA, 35., 2006, João Pessoa. Anais... Jaboticabal: Associação Brasileira de Engenharia Agrícola, 2006. 1 CD-ROM.

ROSENFIELD, G.H.; FITZPATRICK-LINS, K. A coefficient of agreement as a measure of thematic classification accuracy. Photogrammetric Engineering and Remote Sensing, Reston, v.52, n.2, p.223-7, 1986.

RSI. RESEARCH SYSTEMS. User's Guide. ENVI - version 4.0. The Environment for Visualizing Images. 2003. $1.084 \mathrm{p}$.

RUDORFF, C.M.; RIZZI, R.; RUDORFF, B.F.T.; SUGAWARA, L.M.; VIEIRA, C.A.O. Superfícies de reposta espectro-temporal de imagens do sensor MODIS para classificação de áreas de soja no Estado do Rio Grande do Sul. Ciência Rural, Santa Maria, v.37, n.1, p.118-25, 2007.

SCHNEPF R.; DOHLMAN E.; BOLLING C. Argentina \& Brazil sharpen their competitive edge. Special Report in Agricultural Outlook. Economic Research Service/USDA .September 2001. 6 p. Disponível em: www.ers.usda.gov/publications/AgOutlook/sep2001/ao284j.pdf. Acesso em: 26 jul. 2007.

SEAB/DERAL. Secretaria da Agricultura e do Abastecimento do Paraná. Departamento de Economia Rural. Disponível em: www.pr.gov.br/seab. Acesso em: 27 jul. 2007.

SEE, L.M.; FRITZ, S. A method to compare and improve land cover datasets: application to GLC2000 and Modis land cover products. IEEE Transactions on Geoscience and Remote Sensing, New York, v.44, n.7, p.1.740-6, 2006.

STORY, M.; CONGALTON, R.G. Accuracy assessment: a user's perspective. Photogrammetric Engineering and Remote Sensing, Reston, v.52, n.3, p.397-9, 1986.

STERN, A.J.; DORAISWAMY, P.C.; COOK, P.W. Spring wheat classification in an AVHRR image by signature extension from Landsat TM classified images. Photogrammetric Engineering and Remote Sensing, Reston, v.67, n. 2, p.207-11, 2001.

TUCKER, C.; PINZON, J.; BROWN, M.; SLAYBACK, D.; PAK, E.; MAHONEY, R.;

VERMONTE, E.; EL SALEOUS, N. An extended AVHRR 8-km NDVI dataset compatible with MODIS and SPOT vegetation data. International Journal of Remote Sensing, London, v.26, n.20, p.4.485-98, 2005.

XIAO, X.; BOLES, S.; FROLKING, S.; SALAS, W.; MOORE, B.; LI, C.; HE, L.; ZHAO, R. Landscape-scale characterization of cropland in China using VEGETATION sensor data and Landsat TM imagery. International Journal of Remote Sensing, London, v.23, n.18, p.3.579-94, 2002. 